\title{
Remarks on the Theory of Aromatic Free Radicals
}

\author{
Linus Patling and G. W. Wheland, Gates Chemical Laboratory, California Institute of Technology
}

(Received March 5, 1935)

$\mathrm{N}$ the quantum-mechanical treatment of the benzene molecule (and of other aromatic molecules) ${ }^{1}$ the approximation is usually made of neglecting the pairs of electrons which are considered to form bonds in the plane of the ring. The problem then becomes one of discussing the wave function which can be constructed for the remaining electrons by use of the $p_{z}$ orbitals, one for each carbon atom. This discussion can be made either by the molecular orbital method or by the valence bond method, the results being in general agreement. The treatment of a free radical such as phenylmethyl is closely similar, seven $p_{z}$ orbitals and seven electrons being considered for this molecule. It is found convenient in applying the valence bond method $^{2}$ to introduce a "phantom orbital" and an additional electron, a procedure which simplifies the treatment without changing the results.

The quantum-mechanical treatment of the electron affinity of a free radical involves the similar treatment of the negative ion, the wave function for the ion being formed by the use of the same $p_{z}$ orbitals as for the radical, but with one more electron (eight electrons and seven $p_{z}$ orbitals for the phenylmethyl negative ion). The discussion of this problem by the molecular orbital method has been given by Wheland, ${ }^{1}$ who showed that the electron affinity is approximately equal to the difference in coulomb energy of the ion and radical, the difference in resonance energy being small in all the free radicals. This result is not in disagreement with experiment. The valence bond method can also be applied to this problem. For example, we might consider for the phenylmethyl radical (as an approximation)

1 E. Huckel, Zeits. f. Physik 70, 204; 72, 310 (1931); 76, $628(1932) ; 83,632$ (1933); L. Pauling and $G$. W. Wheland, J. Chem. Phys. 1, 362 (1933); L. Pauling and J. Sherman, ibid. 1, 679 (1933); G. W. Wheland, ibid. 2, 474 (1934).

${ }^{2}$ L. Pauling, J. Chem. Phys. 1, 280 (1933), Cf. also L. Pauling and $G$. W. Wheland, reference 1. only the five unexcited structures
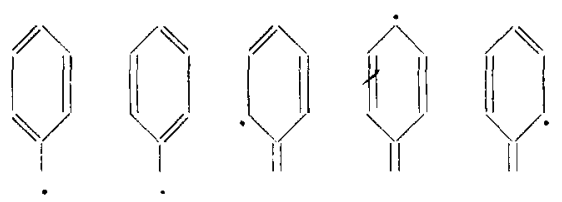

in which the dot represents the odd electron (considered bonded to a phantom orbital in our graphical treatment), and for the ion the five unexcited structures<smiles>Ic1ccccc1</smiles><smiles>c1ccccc1</smiles><smiles>C=C1C=CC=CC1=O</smiles><smiles></smiles><smiles>C=C1C=CC=C[Te]1</smiles>

in which the pair of dots represent two electrons (with opposed spins) occupying the same $p_{2}$ orbital. It is found, however, that the secular equations for the ions involve several integrals which are presumably not small enough to be neglected and for which we have not been able to estimate reliable values; for this reason we have not carried out the calculation. It seems probable, from the form of the canonical structures of radical and ion, that the valence bond method would give essentially the same result as the molecular orbital method, namely, that the difference in resonance energy of radical and ion is small.

The attempted application of the valence bond method in the calculation of the electron affinities of free radicals made by Nilsen ${ }^{3}$ is criticized by Hylleraas in the preceding paper. ${ }^{4}$ We agree with Hylleraas that Nilsen has not correctly evaluated the resonance energy, and in addition we point out that he has neglected to mention the changed coulomb energy, which in our opinion is the major contributor to the electron affinity.

${ }^{3}$ B. Nilsen, J. Chem. Phys. 3, 15 (1935).

4 E. A. Hylleraas, J. Chem. Phys. 3, 313 (1935). 